\title{
Duration of treatment among patients prescribed afatinib or erlotinib as first-line therapy for EGFR mutation-positive non-small-cell lung cancer in the USA
}

\author{
Jonathan Lim*,1, Carl Samuelsen², Amanda Golembesky², Sulena Shrestha ${ }^{3}$, Li Wang ${ }^{3}$ \& \\ Ingolf Griebsch ${ }^{2}$ \\ ${ }^{1}$ Boehringer Ingelheim Pharmaceuticals, Inc., Ridgefield, CT 06877, USA \\ ${ }^{2}$ Boehringer Ingelheim International $\mathrm{GmbH}$, Ingelheim, Germany \\ ${ }^{3}$ STATinMED Research, Plano, TX 75024, USA \\ *Author for correspondence: jonathan.lim@boehringer-ingelheim.com
}

\begin{abstract}
Aim: Evaluate duration of therapy among patients treated with afatinib or erlotinib as first-line therapy for non-small-cell lung cancer (NSCLC). Materials \& methods: NSCLC patients initiating afatinib or erlotinib between 2014 and 2017 were identified in three large claims databases in the USA. Propensity score matching was conducted to compare the duration of treatment between patients by treatment. Results: Patients prescribed afatinib had a significantly longer median duration of treatment compared with those prescribed erlotinib (12.1 vs 9.9 months; $p=0.035)$ and experienced a $14 \%$ reduction in risk of discontinuing therapy (adjusted hazard ratio: 0.86 ; $\mathrm{Cl}: 0.75-0.99$ ). Conclusion: First-line treatment duration in a real-world setting was significantly longer for patients prescribed afatinib compared with erlotinib.
\end{abstract}

First draft submitted: 1 February 2019; Accepted for publication: 26 February 2019; Published online: 19 March 2019

Keywords: afatinib $\bullet$ EGFR tyrosine kinase inhibitors $\bullet$ erlotinib $\bullet$ first-line treatment $\bullet$ non-small-cell lung cancer

EGFR tyrosine kinase inhibitors (TKIs) are standard first-line treatment for patients with EGFR mutation-positive non-small-cell lung cancer (NSCLC) [1,2]. In the USA, there are now three generations of EGFR TKIs approved for the first-line treatment of EGFR mutation-positive NSCLC based on data from randomized controlled trials that have different pharmacological characteristics and mechanisms-of-action: the first-generation reversible TKIs, erlotinib [3,4] and gefitinib [5-7], noncovalently and reversibly bind to EGFR (both wild-type and mutated forms); the second-generation irreversible ErbB family blocker, afatinib [8,9] covalently and irreversibly binds not only to EGFR but also to other members of the ErbB family of receptors, HER2 and HER4 [10,11], as does the EGFR, HER2 and ErbB4 inhibitor, dacomitinib [12]; and the third-generation irreversible EGFR/T790M inhibitor, osimertinib [13], which has low selectivity for wild-type EGFR and high potency toward T790M. EGFR-targeted agents also differ in the mechanisms of acquired resistance that inevitably develop. The most common resistance mechanism to firstand second-generation TKIs is the T790M mutation in exon 20, which has been identified in around $50-70 \%$ of cases [14-17], while the resistance mechanisms for osimertinib appear to be heterogeneous [18].

There have only been two head-to-head trials comparing first- and second-generation EGFR TKIs in EGFR mutation-positive NSCLC and there is no consensus on the preferred first-line EGFR TKI. The Phase IIB LUXLung 7 trial, compared first-line afatinib with gefitinib in EGFR mutation-positive (Del19 or L858R) NSCLC and was the first prospective, randomized trial to compare first- and second-generation TKIs. LUX-Lung 7 showed that afatinib prolonged progression-free survival (PFS; hazard ratio [HR]: $0.73 ; 95 \% \mathrm{CI}: 0.57-0.95 ; \mathrm{p}=0.017$ ) and time to treatment failure (TTF; HR: 0.73; 95\% CI: 0.58-0.92; $\mathrm{p}=0.0073$ ) versus the first-generation EGFR TKI gefitinib [19]; data for the co-primary end point of overall survival (OS) are currently immature [19]. More recently, in a study population that excluded patients with metastases in the central nervous system, the Phase III ARCHER 1050 trial compared the second-generation EGFR TKI dacomitinib with gefitinib and showed that dacomitinib significantly prolonged PFS compared with gefitinib (HR: 0.59; $\mathrm{p}<0.0001$ ) [12]; exploratory 
analysis showed improvement in OS (HR: 0.76; 95\% CI: 0.58-0.99) [20]. Taken together, this evidence suggests that second-generation EGFR TKIs may be more effective than first-generation TKIs. Although no randomized controlled clinical trial has compared afatinib with erlotinib as first-line treatment in EGFR mutation-positive NSCLC, several network meta-analyses have been conducted to evaluate this comparison using slightly different assumptions [21-24]; results have suggested superior PFS with afatinib compared with erlotinib in patients with common EGFR mutations [21] or shown no statistically significant differences between treatments [22-24].

While randomized controlled trials are conducted to assess the efficacy and safety of treatments in well-defined, controlled clinical conditions and selected study populations, trial outcomes may not always reflect real-world experience. Patients in clinical practice may, for instance, present with different risk factor profiles than those included in controlled clinical trials, making it harder to predict whether results from controlled trials will apply to the real-world patient populations [25]. For example, in oncology, real-world effectiveness estimates based on surrogate end points have been shown to be lower than efficacy estimates from randomized controlled trials [26]. Real-world studies can complement data from randomized controlled trials by providing information on treatment use in everyday practice in a population of patients that might otherwise have been excluded from participation in controlled trials. Real-world evidence can also be used to provide insights into treatment comparisons that have not been studied in a controlled trial setting. Currently, limited data are available on the use of EGFR TKIs in everyday clinical practice. Retrospective analysis of real-world outcomes in small, single-center patient populations have shown improved survival outcomes with second-generation compared with first-generation agents in Canada [27] and favorable PFS with afatinib compared with erlotinib and gefitinib in Japan and Korea [28,29].

To address these gaps in the literature and provide insights into the comparative effectiveness of afatinib and erlotinib, this study assessed time on treatment with these agents in the real-world setting. The study included patients with NSCLC who initiated first-line treatment with afatinib or erlotinib, and used data from three administrative claims databases in the USA.

\section{Methods}

Data source

This study pooled data retrieved from three large, administrative claims databases in the USA: Truven MarketScan ${ }^{\circledR}$ (spanning 1 January 2013-31 March 2017) [30], IMS PharMetrics Plus (spanning 1 January 2013-30 September 2017) [31] and Optum Clinformatics ${ }^{\top \mathrm{TM}}$ Data Mart (spanning 1 January 2013-30 September 2017) [32]. The pooled data included patient demographics, enrolment history, and medical and pharmacy claims for more than 110 million members of commercial plans and Medicare Advantage/supplemental plans. MarketScan includes complete, person-specific information on outpatient prescriptions from employer-provided health insurance and Medicaid. IMS PharMetrics Plus claims database includes claims for medical (provider and institutional) and pharmacy services, with claims paid and allowed amounts, as well as all-patient payment amounts. Optum Clinformatics ${ }^{\top M}$ Data Mart database is a single-payer source of claims data that also includes claims for commercially insured and Medicare Advantage affiliate members with linked health risk assessment and socioeconomic status data. Given the possibility that one patient could be insured by two different plans and to avoid duplication, duplicate patient information among the three data sources were identified using a research algorithm employed in previous studies [33-35]. The research algorithm used age, sex, US region, hospital admission date, discharge date and primary discharge diagnosis to identify duplicate patients; only patients with the most complete records from one database were retained for analysis. As the databases used for this study do not provide an explicit indicator of death for privacy reasons, we used a previously developed algorithm to define death [36-38]. Analyses were also performed for each database population separately.

\section{Sample selection \& construction}

Patients were required to have initiated EGFR TKI monotherapy with afatinib or erlotinib during the identification period (1 July 2013 to 28 February 2017 [Truven MarketScan] or 31 August 2017 [IMS PharMetrics Plus and Optum Clinformatics ${ }^{\text {TM }}$ Data Mart]); the initiation date of EGFR TKI therapy was used as the index date. Monotherapy was defined as no other systemic therapy prescription fills within 30 days after the index date. Furthermore, patients needed to be aged $\geq 18$ years on the index date and have at least one inpatient or outpatient claim with a diagnosis of lung cancer (International Classification of Diseases, Ninth Revision, Clinical Modification [ICD-9-CM] codes 162.x or ICD-10-CM: C34.xx) during the 30-day pre-index period or within 30 days after the index date. 
Patients were required to have had continuous health plan enrolment with medical and pharmacy benefits for $\geq 6$ months pre-index date (baseline) and $\geq 30$ days post-index date. Patients with $\geq 1$ prescription claim for other systemic NSCLC treatment during the baseline period were excluded, unless the treatment was a chemotherapy regimen accompanied with a code for lung surgery. Details of these are given in the Supplementary Methods. Patients were stratified into two cohorts based on the index drug: the afatinib cohort or the erlotinib cohort. Patients identified in 2013 (from July to December) were excluded from the primary analysis to reduce channelling bias arising from selecting patients in the same year that afatinib was approved (July 2013) by the US FDA. This approach to minimizing channelling bias has often been used previously [39-41].

\section{Baseline characteristics}

Patient demographic and clinical characteristics assessed at the index date included age, sex, geographic region, index year, Deyo-modified Charlson Comorbidity Index (CCI) Score $[42,43]$ and comorbidities (cerebrovascular disease [44], coronary heart disease and myocardial infarction [45], heart failure and peripheral arterial disease [46], diabetes mellitus [47], chronic kidney disease [48], liver disease [49] and chronic obstructive pulmonary disease [COPD] [50]).

\section{Propensity score matching}

Patients in the afatinib and erlotinib cohorts were matched 1:1 using propensity scores to account for observable differences in baseline variables between cohorts. Propensity scores were calculated using a logistic regression; covariates included age, sex, geographic region, Deyo-modified CCI score and individual comorbidities. The matching algorithm selected the closest matches between cohorts, where propensity scores were within \pm 0.001 units of each other.

\section{Outcomes}

The primary outcome was duration of first-line treatment, calculated as time from the index date to the end of treatment. End of treatment was defined as either date of treatment discontinuation (>60-day gap in supply of the index drug from the last day of supply) or the addition of a chemotherapy treatment before discontinuation of the index medication, whichever came first. Patients whose treatments were used until the disenrollment in the healthcare plan, or end of the study period were censored at the end of follow-up [51]. Duration of second-line therapy was a secondary outcome. Second-line therapy was considered to have begun on the date of the first prescription fill for a systemic therapy after end of first-line therapy and included all agents prescribed in the first 30 days after initiation of second-line therapy. Duration of second-line therapy was defined in the same way as duration of first-line therapy.

\section{Statistical analyses}

Baseline characteristics in the afatinib and erlotinib cohorts were summarized descriptively using mean values, medians, ranges and standard deviations of continuous variables and frequency distributions for categorical variables. Baseline characteristics for unmatched and matched cohorts were compared using t-tests for continuous variables and $\chi^{2}$ tests for categorical variables. In addition to $\mathrm{p}$-values, standardized differences were calculated for each variable compared between the cohorts. The balance of covariates was checked based on standardized differences using a threshold of $10 \%$.

For the unmatched cohorts, Cox proportional hazard models were used to assess whether differences in therapy duration between cohorts were associated with cohort assignment. Covariates included in the models included age, sex, US region, baseline CCI score and baseline comorbidities.

Duration of treatment was compared between the matched afatinib and erlotinib cohorts using Kaplan-Meier analyses, with log-rank tests to determine a difference between these survival curves; p-values were reported. One month was defined as 30 days.

Only patients with complete information were included in the analysis of data for second-line therapy; patients were deemed to have incomplete information if they were lost to follow-up or were censored at the end of the study period. This was a retrospective study with no a priori hypothesis testing; therefore, we did not undertake a formal calculation of sample size and statistical power. All analyses were conducted using SAS, version 9.3 (SAS Institute, NC, USA). 


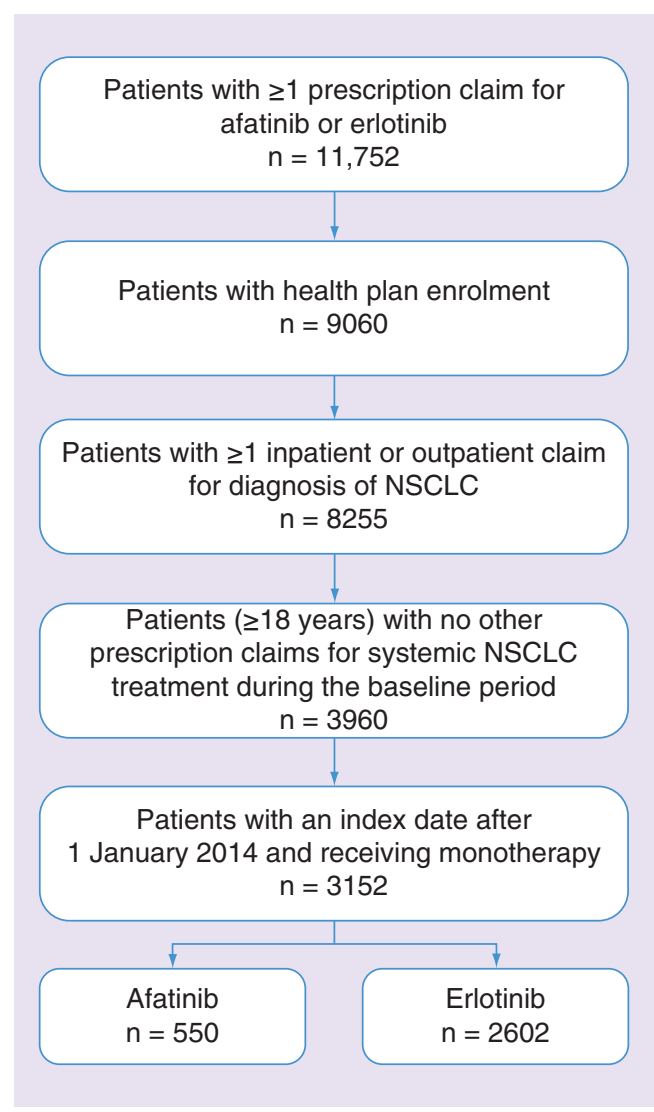

Figure 1. Sample selection.

NSCLC: Non-small-cell lung cancer.

\section{Results}

Overall, 3152 patients with NSCLC met the study criteria: 550 patients who initiated treatment with afatinib and 2602 patients who initiated treatment with erlotinib (Figure 1). Before propensity matching, there were differences in several baseline characteristics between the two treatment cohorts; patients prescribed afatinib were younger (63.3 vs 67.1 years; $p<0.001)$ and had greater mean Deyo-modified CCI score $(8.5$ vs $8.1 ; p=0.002)$ than those prescribed erlotinib. Patients prescribed afatinib were also less likely to have comorbid cerebrovascular disease (10.9 vs $16.5 \% ; \mathrm{p}=0.001)$, peripheral arterial disease ( 8.0 vs $11.3 \% ; \mathrm{p}=0.022)$, chronic kidney disease (5.8 vs $8.5 \%$; $\mathrm{p}=0.038$ ) or COPD (31.6 vs 37.4\%; $\mathrm{p}=0.010$ ), than those prescribed erlotinib (see Table 1 ).

For the unmatched cohorts, after adjusting for co-variates, patients prescribed afatinib as the first-line monotherapy had significantly longer duration on first-line treatment compared with those prescribed erlotinib as first-line therapy (adjusted HR: 0.86; 95\% CI: 0.75-0.99; Figure 2; median duration of treatment: afatinib 12.0 months vs erlotinib 9.9 months). Male gender, presence of COPD and presence of chronic kidney disease were factors associated with a significantly shorter duration of first-line therapy (see Figure 2). There was also regional variation in duration of first-line therapy.

Propensity score matching of the afatinib and erlotinib patients yielded 525 matched pairs with well-balanced baseline characteristics (Table 1).

After matching, patients prescribed afatinib had a significantly longer median duration of treatment compared with those prescribed erlotinib (12.1 vs 9.9 months; $p=0.035$; Figure $3 \mathrm{~A}$ ). Data from individual databases were consistent with that of the pooled analysis (Supplementary Figure 1).

Among patients with follow-up after discontinuation of first-line therapy ( $\mathrm{n}=222$ for afatinib and $\mathrm{n}=275$ for erlotinib), $66 \%$ of patients in the afatinib cohort and $67 \%$ of patients in the erlotinib cohort had evidence of receiving a second-line therapy. Details of second-line treatments are shown in Table 2; in both cohorts, monotherapy TKI treatment was the most prescribed second-line treatment (afatinib 37\%; erlotinib 35\%) followed by pemetrexedbased (12\% in both cohorts). Duration of second-line therapy varied between cohorts (afatinib: 8.0 months vs erlotinib: 5.7 months), although this did not reach statistical significance $(\mathrm{p}=0.057)$ (Figure 3B). 
Table 1. Baseline characteristics among the cohort of patients treated with afatinib and erlotinib before and after propensity score matching.

\begin{tabular}{|c|c|c|c|c|c|c|c|c|}
\hline \multirow{2}{*}{$\begin{array}{l}\text { Demographic and clinical } \\
\text { characteristics }\end{array}$} & \multicolumn{3}{|c|}{ Before matching } & \multirow[t]{2}{*}{ STD } & \multicolumn{3}{|c|}{ After matching } & \multirow[t]{2}{*}{ STD } \\
\hline & $\begin{array}{l}\text { Erlotinib } \\
(\mathrm{N}=2602)\end{array}$ & $\begin{array}{l}\text { Afatinib } \\
(\mathrm{N}=550)\end{array}$ & p-value & & $\begin{array}{l}\text { Erlotinib } \\
(\mathrm{N}=525)\end{array}$ & $\begin{array}{l}\text { Afatinib } \\
(\mathrm{N}=525)\end{array}$ & p-value & \\
\hline Age, years, mean $\pm S D$ & $67.1(12.0)$ & $63.3(11.4)$ & $<0.001$ & 32.3 & $64(11.7)$ & $63.8(11.3)$ & 0.805 & 1.5 \\
\hline \multicolumn{9}{|l|}{ Age category; $\mathrm{n}(\%)$ : } \\
\hline$-18-34$ years & $8(0.3)$ & $0(0.0)$ & 0.193 & 7.9 & $0(0.0)$ & $0(0.0)$ & $\mathrm{N} / \mathrm{A}$ & $\mathrm{N} / \mathrm{A}$ \\
\hline$-35-49$ years & $149(5.7)$ & $57(10.4)$ & $<0.001$ & 17.1 & $47(9.0)$ & $49(9.3)$ & 0.830 & 1.3 \\
\hline$-50-54$ years & $221(8.5)$ & $71(12.9)$ & 0.001 & 14.3 & $63(12.0)$ & $66(12.6)$ & 0.778 & 1.7 \\
\hline$-55-59$ years & $336(12.9)$ & $89(16.2)$ & 0.041 & 9.3 & $87(16.6)$ & $81(15.4)$ & 0.614 & 3.1 \\
\hline$-60-64$ years & $501(19.3)$ & $104(18.9)$ & 0.852 & 0.9 & $113(21.5)$ & $101(19.2)$ & 0.358 & 5.7 \\
\hline$-\geq 65$ years & $1387(53.3)$ & $229(41.6)$ & $<0.001$ & 23.5 & $215(41.0)$ & $228(43.4)$ & 0.417 & 5.0 \\
\hline \multicolumn{9}{|l|}{ Sex; n (\%): } \\
\hline - Male & $921(35.4)$ & $195(35.5)$ & 0.979 & 0.1 & $179(34.1)$ & $184(35.0)$ & 0.746 & 2.0 \\
\hline - Female & $1681(64.6)$ & $355(64.5)$ & 0.979 & 0.1 & $346(65.9)$ & $341(65.0)$ & 0.746 & 2.0 \\
\hline \multicolumn{9}{|l|}{ US region; $n(\%)$ : } \\
\hline - Northeast & $525(20.2)$ & $139(25.3)$ & 0.008 & 12.2 & $128(24.4)$ & $126(24.0)$ & 0.885 & 0.9 \\
\hline - North-central & $560(21.5)$ & $100(18.2)$ & 0.080 & 8.4 & $97(18.5)$ & 99 (18.9) & 0.874 & 1.0 \\
\hline - South & $866(33.3)$ & $224(40.7)$ & 0.001 & 15.5 & $212(40.4)$ & $213(40.6)$ & 0.950 & 0.4 \\
\hline - West & $624(24.0)$ & $87(15.8)$ & $<0.001$ & 20.5 & $88(16.8)$ & $87(16.6)$ & 0.934 & 0.5 \\
\hline - Unknown & $27(1.0)$ & $0(0.0)$ & 0.016 & 14.5 & $0(0.0)$ & $0(0.0)$ & $\mathrm{N} / \mathrm{A}$ & $\mathrm{N} / \mathrm{A}$ \\
\hline $\begin{array}{l}\text { Deyo-modified CCI Score, } \\
\text { mean } \pm \text { SD }\end{array}$ & $8.1(3.0)$ & $8.5(2.5)$ & 0.002 & 13.9 & $8.3(2.4)$ & $8.5(2.5)$ & 0.267 & 6.9 \\
\hline \multicolumn{9}{|l|}{ Individual comorbidities; $\mathrm{n}(\%)$ : } \\
\hline - Cerebrovascular disease & $429(16.5)$ & $60(10.9)$ & 0.001 & 16.3 & $53(10.1)$ & $60(11.4)$ & 0.486 & 4.3 \\
\hline - Coronary heart disease & $373(14.3)$ & $71(12.9)$ & 0.382 & 4.2 & $64(12.2)$ & $67(12.8)$ & 0.779 & 1.7 \\
\hline - Myocardial infarction & $108(4.2)$ & $16(2.9)$ & 0.174 & 6.7 & $10(1.9)$ & $16(3.0)$ & 0.233 & 7.4 \\
\hline - Heart failure & $204(7.8)$ & $30(5.5)$ & 0.053 & 9.6 & $23(4.4)$ & $29(5.5)$ & 0.393 & 5.3 \\
\hline - Peripheral arterial disease & $295(11.3)$ & $44(8.0)$ & 0.022 & 11.3 & $39(7.4)$ & $44(8.4)$ & 0.567 & 3.5 \\
\hline - Diabetes mellitus & $520(20.0)$ & $96(17.5)$ & 0.174 & 6.5 & $67(12.8)$ & $88(16.8)$ & 0.068 & 11.3 \\
\hline - Chronic kidney disease & $220(8.5)$ & $32(5.8)$ & 0.038 & 10.3 & $27(5.1)$ & $32(6.1)$ & 0.503 & 4.1 \\
\hline - Liver disease & $453(17.4)$ & 93 (16.9) & 0.778 & 1.3 & 94 (17.9) & $86(16.4)$ & 0.512 & 4.0 \\
\hline - COPD & 974 (37.4) & 174 (31.6) & 0.010 & 12.2 & $172(32.8)$ & $168(32.0)$ & 0.792 & 1.6 \\
\hline
\end{tabular}

CCI: Charlson Comorbidity Index; COPD: Chronic obstructive pulmonary disease; NSCLC: Non-small-cell lung cancer; SD: Standard deviation; STD: Standardized difference.

\section{Discussion}

This study assessed duration of first-line treatment with afatinib versus erlotinib in a large cohort of patients with NSCLC treated in the USA. After propensity score matching, results showed that patients prescribed afatinib had a longer median treatment duration and lower risk of discontinuing first-line treatment than patients prescribed erlotinib. Discontinuation of first-line therapy may result from a range of reasons, not limited to disease progression or tolerability issues. The results suggest that first-generation and second-generation TKIs are not interchangeable and, although the reasons for discontinuation were not evaluated in this study, it can be hypothesized that longer treatment duration may reflect improved benefit for patients treated with afatinib compared with erlotinib in clinical practice, resulting from the higher potency and broader irreversible ErbB blockade of afatinib compared with erlotinib, which inhibits EGFR alone [10,11]. For example, inhibition of both ErbB2 and ErbB3 by afatinib has been implicated in the acquired resistance to first-generation TKIs [52,53], suggesting that afatinib has the potential to delay resistance mechanisms compared with erlotinib. Afatinib has also been shown to have modest activity in patients with acquired resistance to first-generation TKIs [54-56].

As a real-world study, this analysis provides data on outcomes in a broader range of patients than those assessed in previous clinical trials of afatinib or erlotinib. Rates of comorbidity are not commonly reported in controlled clinical trials, as such it is especially notable that rates of comorbidity in the patients reported here were high, 


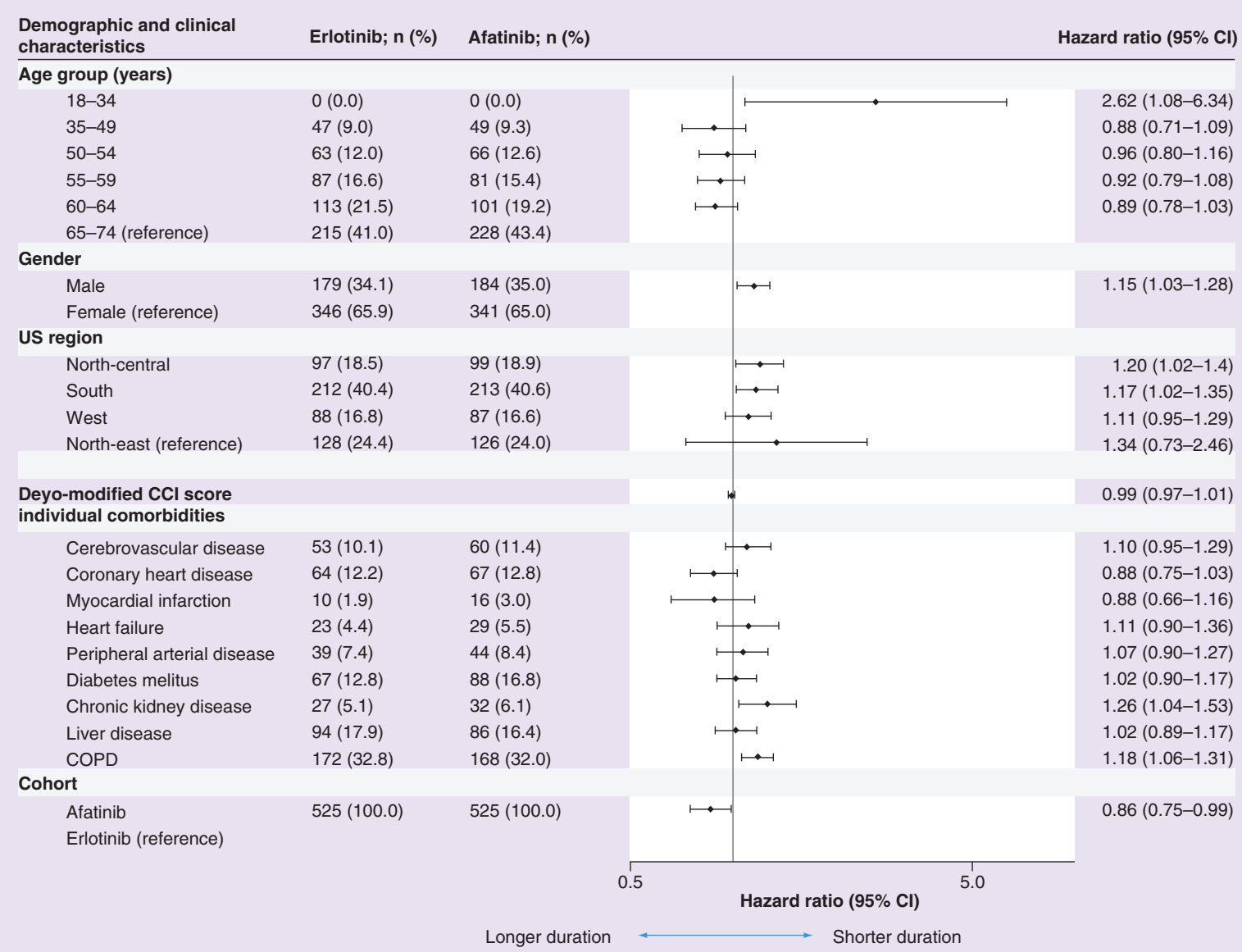

Figure 2. Multivariable-adjusted Cox proportional hazard models for duration of first-line treatment in the unmatched cohort. CCl: Charlson Comorbidity Index; COPD: Chronic obstructive pulmonary disease.

Table 2. Second-line treatment patterns among the cohort of patients treated with afatinib and erlotinib after propensity score matching.

\begin{tabular}{|c|c|c|}
\hline N (\%) & $\begin{array}{l}\text { Erlotinib } \\
n=275\end{array}$ & $\begin{array}{l}\text { Afatinib } \\
n=222\end{array}$ \\
\hline Second-line therapy & $184(66.9 \%)$ & $146(65.8 \%)$ \\
\hline Doublets & $6(2.2 \%)$ & $1(0.5 \%)$ \\
\hline Pemetrexed-based regimens & $33(12.0 \%)$ & $26(11.7 \%)$ \\
\hline Pemetrexed + bevacizumab-based regimens & $9(3.3 \%)$ & $9(4.1 \%)$ \\
\hline Bevacizumab-based regimens & $4(1.5 \%)$ & $2(0.9 \%)$ \\
\hline Single-agent chemotherapy & $4(1.5 \%)$ & $3(1.4 \%)$ \\
\hline Immunotherapies & $15(5.5 \%)$ & $9(4.1 \%)$ \\
\hline Other TKIs: & $96(34.9 \%)$ & $81(36.5 \%)$ \\
\hline - Afatinib & $37(13.5 \%)$ & $15(6.8 \%)$ \\
\hline - Erlotinib & $24(8.7 \%)$ & $30(13.5 \%)$ \\
\hline - Gefitinib & $1(0.4 \%)$ & $7(3.2 \%)$ \\
\hline - Osimertinib & $34(12.4 \%)$ & $29(13.1 \%)$ \\
\hline - Other drugs & $17(6.2 \%)$ & $15(6.8 \%)$ \\
\hline
\end{tabular}


(A)

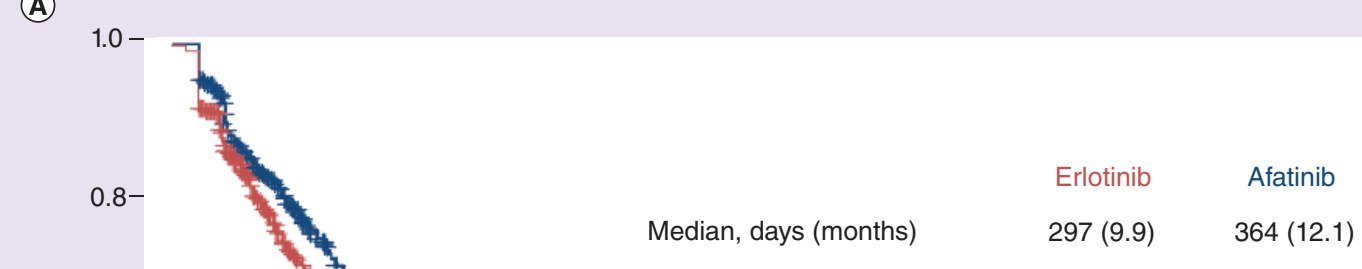

$0.4-$

$0.2-$

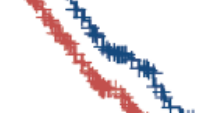

$297(9.9) \quad 364(12.1)$
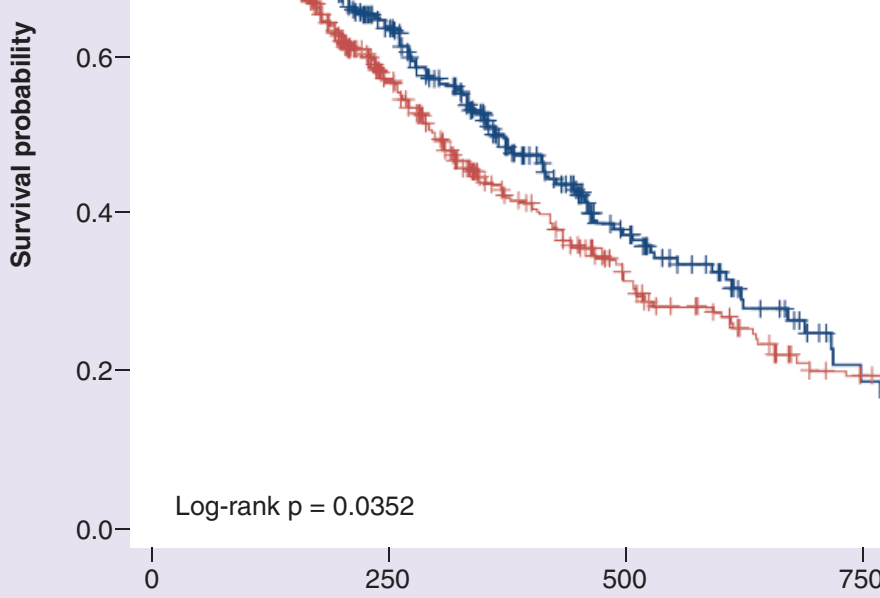

1000

1250

Time (days)

Afatinib

(B)
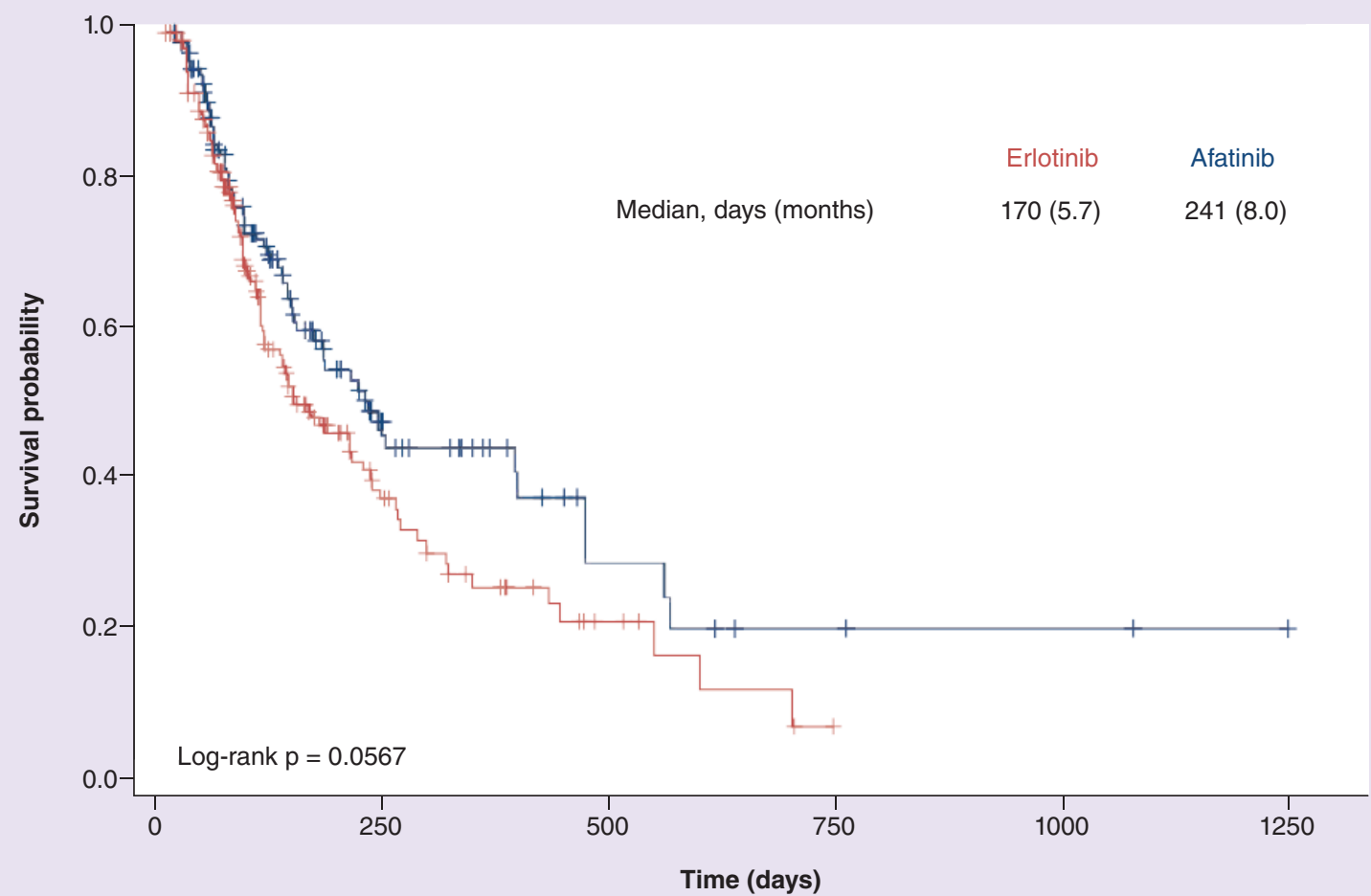

Afatinib 
highlighting that patients typically seen in everyday clinical care do frequently have comorbid disease that may preclude their inclusion in controlled trials.

There is limited published research on first-line treatment duration of afatinib compared with erlotinib in patients with EGFR mutation-positive NSCLC in the real-world setting in the USA. TTF, defined as the time from the first dose to the last dose of treatment, is synonymous to time on treatment as reported here. In a Japanese chart review, analysis of data from approximately 1300 patients with EGFR mutation-positive NSCLC who received EGFR-TKI therapy showed longer TTF with afatinib (14.1 months) compared with erlotinib (10.6 months) or gefitinib (10.6 months) [57]; there was also a trend towards OS benefit. In data from two retrospective, observational multicenter studies (the GioTag study and the RealGiDo study), time on treatment with afatinib was 11.9 months (90\% CI: 10.9-12.2) [58] and 18.7 months (95\% CI: 15.1-21.5) [59], respectively. A retrospective, claims-based analysis supplemented with data from medical chart review reported a treatment duration with erlotinib of 7.9 months [60]. Although these studies differ with respect to study design, methods, patient population and data collection, it is reassuring that the data reported here are consistent with other real-world studies and demonstrate that afatinib is an effective first-line treatment for patients with EGFR mutation-positive NSCLC in clinical practice.

There are also only sparse published data on comorbidities in patients with EGFR mutation-positive NSCLC; as such, our data provide an interesting snapshot of common comorbidities in this patient population. In the total enrolled patient population, before propensity score matching, COPD was the most common comorbidity in both treatment groups, affecting approximately a third of patients, followed by diabetes mellitus and kidney disease. It should also be noted that, before propensity score matching, there were a number of significant differences in rates of comorbidity between the afatinib and erlotinib cohorts: the erlotinib cohort generally had more comorbidity than the afatinib cohort. This may potentially reflect differences in the perceived tolerability profile between treatments, with clinicians being more likely to prescribe erlotinib as a treatment in patients with a high comorbidity burden due to a perception of greater tolerability.

In our study, approximately $65 \%$ of patients in both treatment groups went on to receive subsequent therapy, although it should be noted that our analysis of second-line treatment excluded patients who were lost to follow-up (due to health plan disenrollment or censoring) and may underestimate the number of patients receiving second-line therapy. Our results are encouraging and suggest that that this real-world USA population remained fit enough to receive subsequent treatment therapies. To provide broad context to our data, in Phase III trials of first-generation EGFR TKIs, rates of subsequent therapy were around 60-70\% [4,61,62]. Analysis of the LUX-Lung 3, 6 and 7 trials showed that $71 \%$ of patients received a further line of treatment following discontinuation of afatinib; subsequent therapy rates were also higher (88\%) for the subgroup of patients from countries with a universal reimbursement system [63]. Similar to data reported here, subsequent therapy after afatinib in controlled trials predominantly consisted of platinum-based chemotherapy, with other therapies received including single-agent chemotherapy, firstgeneration EGFR TKI monotherapy, chemotherapy combination therapies and third-generation EGFR TKIs [15].

It should be noted that, for a proportion of our study period, there were no approved treatment options for patients that progressed on an EGFR TKI, which likely explains why such a diverse range of second-line treatments were recorded. Osimertinib received accelerated approval in the USA in November 2015, in the middle of our study period, and remains the only approved treatment in patients with advanced NSCLC that have progressed after treatment with EGFR TKI. As such, current second-line treatment practice likely differs from our findings. In a recent single-center retrospective analysis, all patients $(n=49)$ progressing on afatinib who had T790M mutations received subsequent therapy with osimertinib [15]. As resistance mechanisms (e.g., acquired resistance caused by EGFR T790M mutation) could not be evaluated in our analysis of second-line treatment, it is unclear how specific resistance mechanisms may have influenced treatment choice in clinical practice. It is possible that high rates of second-line afatinib use in the erlotinib cohort may reflect findings from the LUX-Lung 1 study that showed a PFS benefit with afatinib compared with placebo in this patient population [54]. Further research is needed to assess the optimal sequence of therapy with EGFR TKIs in individual patients over the course of their treatment now a targeted second-line treatment option is available.

To our knowledge, this is the first real-world claims-based study in the USA to compare the first-line use of afatinib to erlotinib in patients with NSCLC. Strengths of the study include the large sample size and use of data from three administrative claims databases to overcome challenges related to data scarcity. Use of several databases strengthens the data by providing a broader sample of patients, although older patients and patients of low socioeconomic status are known to be under-represented in administrative claims databases; this is a limitation. 
The use of propensity score matching ensured that the samples were balanced for analysis and this finding was confirmed by analysis of data from the unmatched population using Cox multivariate analysis.

An important consideration when interpreting the findings of this study is that the EGFR mutational status of patients was not known, as data on EGFR mutation status is not captured in administrative claims data. As both treatments evaluated in this study are only approved for use as first-line treatment in patients with confirmed EGFR mutation-positive NSCLC, receipt of these treatments was used as a proxy for EGFR mutation-positive NSCLC. There are also several additional limitations inherent to retrospective observational studies using claims data that should be considered when interpreting the findings reported. Administrative claims data only contain diagnostic and procedure codes recorded for reimbursement purposes, and so may not include information on certain clinical and disease-specific parameters that could influence outcome. Data can be subject to coding errors or data omissions, which should be considered when interpreting the findings. For example, the accuracy of the coding may be influenced by reimbursed treatment options for patients, setting of clinical care and additional procedures or laboratory work necessary to rule out potential diagnoses. In addition, information on inpatient treatments may not be systematically captured. Another consideration is that it is possible that patients did not take the prescribed medications directly after filling their prescription; as such, treatment initiation dates and subsequent duration of treatment analyses are approximate. Selection bias can result in analysis of claims data from the requirement for patients to have continuous health plan enrolment to be included in analysis. Another bias is the absence of clear mortality data in commercial databases and the consequent use of algorithms to determine death; as mortality data was used in censoring as well as calculation of number of patients receiving subsequent therapies, these values may be prone to misclassification bias.

In conclusion, this claims-based study suggests that patients treated with afatinib as first-line monotherapy may have a longer first-line treatment duration than patients treated with erlotinib. Future studies are warranted to identify the reasons underlying this observation.

\section{Summary points}

- Evidence suggests that as first-line treatment in EGFR mutation-positive non-small-cell lung cancer (NSCLC), second-generation EGFR tyrosine kinase inhibitors (TKIs) may be more effective than first-generation TKIs, although no randomized controlled clinical trial has compared afatinib with erlotinib in this setting.

- Real-world studies can complement data from randomized controlled trials by providing information on treatment use in everyday practice in a population of patients that might otherwise have been excluded from participation in controlled trials; there is currently limited data on the use of EGFR TKIs in everyday clinical practice.

- This study was an analysis of patients who initiated first-line treatment with afatinib or erlotinib in three large, administrative claims databases in the USA; the primary outcome was duration of first-line treatment with each agent.

- Propensity score matching of the afatinib and erlotinib patients yielded 525 matched pairs with well-balanced baseline characteristics.

- Patients prescribed afatinib had a significantly longer median duration of treatment compared with those prescribed erlotinib (12.1 vs 9.9 months); it can be hypothesized that longer treatment duration may reflect improved benefit for patients treated with afatinib compared with erlotinib in clinical practice.

\section{Author contribution}

The authors were fully responsible for all content and editorial decisions, were involved at all stages of manuscript development and have approved the final version of the manuscript.

Financial \& competing interests disclosure

This study was supported by Boehringer Ingelheim. J Lim, C Samuelsen, A Golembesky and I Griebsch are employees of Boehringer Ingelheim. S Shrestha and L Wang are employees of SIMR, and received consultancy fees from Boehringer Ingelheim for this work. The authors have no other relevant affiliations or financial involvement with any organization or entity with a financial interest in or financial conflict with the subject matter or materials discussed in the manuscript apart from those disclosed.

Medical writing assistance, supported financially by Boehringer Ingelheim, was provided by S Patel during the preparation of this article. 
Open access

This work is licensed under the Attribution-NonCommercial-NoDerivatives 4.0 Unported License. To view a copy of this license, visit http://creativecommons.org/licenses/by-nc-nd/4.0/

\section{References}

Papers of special note have been highlighted as: $\bullet$ of interest; $\bullet \bullet$ of considerable interest

1. Planchard D, Popat S, Kerr K et al. Metastatic non-small cell lung cancer: ESMO Clinical Practice Guidelines for diagnosis, treatment and follow-up. Ann. Oncol. 29(Suppl. 4), iv192-iv237 (2018).

2. NCCN Clinical Practice Guidelines in Oncology. Non-Small Cell Lung Cancer. Version 1-2019 (2018). www.nccn.org/professionals/physician_gls/pdf/nscl.pdf

3. Rosell R, Carcereny E, Gervais R et al. Erlotinib versus standard chemotherapy as first-line treatment for European patients with advanced EGFR mutation-positive non-small-cell lung cancer (EURTAC): a multicentre, open-label, randomised Phase III trial. Lancet Oncol. 13(3), 239-246 (2012).

4. Zhou C, Wu YL, Chen G et al. Erlotinib versus chemotherapy as first-line treatment for patients with advanced EGFR mutation-positive non-small-cell lung cancer (OPTIMAL, CTONG-0802): a multicentre, open-label, randomised, Phase III study. Lancet Oncol. 12(8), 735-742 (2011).

5. Maemondo M, Inoue A, Kobayashi K et al. Gefitinib or chemotherapy for non-small-cell lung cancer with mutated EGFR. N. Engl. J. Med. 362(25), 2380-2388 (2010).

6. Mitsudomi T, Morita S, Yatabe Y et al. Gefitinib versus cisplatin plus docetaxel in patients with non-small-cell lung cancer harbouring mutations of the epidermal growth factor receptor (WJTOG3405): an open label, randomised Phase III trial. Lancet Oncol. 11(2), $121-128$ (2010).

7. Mok TS, Wu YL, Thongprasert S et al. Gefitinib or carboplatin-paclitaxel in pulmonary adenocarcinoma. N. Engl. J. Med. 361(10), 947-957 (2009).

8. Sequist LV, Yang JC, Yamamoto N et al. Phase III study of afatinib or cisplatin plus pemetrexed in patients with metastatic lung adenocarcinoma with EGFR mutations. J. Clin. Oncol. 31(27), 3327-3334 (2013).

9. Wu YL, Zhou C, Hu CP et al. Afatinib versus cisplatin plus gemcitabine for first-line treatment of Asian patients with advanced non-small-cell lung cancer harbouring EGFR mutations (LUX-Lung 6): an open-label, randomised Phase III trial. Lancet Oncol. 15(2), 213-222 (2014).

- Phase III ARCHER 1050 study showing that the second-generation EGFR tyrosine kinase inhibitor (TKI), dacomitinib, was more effective than the first-generation TKI, gefitinib, as first-line treatment of EGFR mutation-positive non-small-cell lung cancer (NSCLC).

10. Li D, Ambrogio L, Shimamura T et al. BIBW2992, an irreversible EGFR/HER2 inhibitor highly effective in preclinical lung cancer models. Oncogene 27(34), 4702-4711 (2008).

11. Solca F, Dahl G, Zoephel A et al. Target binding properties and cellular activity of afatinib (BIBW 2992), an irreversible ErbB family blocker. J. Pharmacol. Exp. Ther. 343(2), 342-350 (2012).

12. Wu YL, Cheng Y, Zhou X et al. Dacomitinib versus gefitinib as first-line treatment for patients with EGFR-mutation-positive non-small-cell lung cancer (ARCHER 1050): a randomised, open-label, Phase III trial. Lancet Oncol. 18(11), 1454-1466 (2017).

13. Soria JC, Ohe Y, Vansteenkiste J et al. Osimertinib in untreated EGFR-mutated advanced non-small-cell lung cancer. N. Engl. J. Med. 378(2), 113-125 (2018).

14. Arcila ME, Oxnard GR, Nafa K et al. Rebiopsy of lung cancer patients with acquired resistance to EGFR inhibitors and enhanced detection of the T790M mutation using a locked nucleic acid-based assay. Clin. Cancer Res. 17(5), 1169-1180 (2011).

15. Hochmair MJ, Buder A, Schwab S et al. Liquid-biopsy-based identification of EGFR T790M mutation-mediated resistance to afatinib treatment in patients with advanced EGFR mutation-positive NSCLC, and subsequent response to osimertinib. Target Oncol. 14(1), 75-83 (2018).

16. Sequist LV, Waltman BA, Dias-Santagata D et al. Genotypic and histological evolution of lung cancers acquiring resistance to EGFR inhibitors. Sci. Transl. Med. 3(75), 75ra26 (2011).

17. Wu SG, Liu YN, Tsai MF et al. The mechanism of acquired resistance to irreversible EGFR tyrosine kinase inhibitor-afatinib in lung adenocarcinoma patients. Oncotarget 7(11), 12404-12413 (2016).

18. Ramalingam SS, Yang JC, Lee CK et al. Osimertinib as first-line treatment of EGFR mutation-positive advanced non-small-cell lung cancer. J. Clin. Oncol. 36(9), 841-849 (2018).

19. Park K, Tan EH, O’Byrne K et al. Afatinib versus gefitinib as first-line treatment of patients with EGFR mutation-positive non-small-cell lung cancer (LUX-Lung 7): a Phase IIB, open-label, randomised controlled trial. Lancet Oncol. 17(5), 577-589 (2016).

-• Primary analysis of the LUX-Lung 7 trial, the first head-to-head trial comparing a first-generation TKI (gefitinib) with a second-generation ERBB family blocker (afatinib) as first-line treatment of EGFR mutation-positive NSCLC and illustrating improved progression-free survival (PFS) with afatinib. 
20. Mok TS, Cheng Y, Zhou X et al. Improvement in overall survival in a randomized study that compared dacomitinib with gefitinib in patients with advanced non-small-cell lung cancer and EGFR-activating mutations. J. Clin. Oncol. 36(22), 2244-2250 (2018).

21. Popat S, Mok T, Yang JC et al. Afatinib in the treatment of EGFR mutation-positive NSCLC-a network meta-analysis. Lung Cancer 85(2), 230-238 (2014).

22. Haaland B, Tan PS, De Castro G Jr, Lopes G. Meta-analysis of first-line therapies in advanced non-small-cell lung cancer harboring EGFR-activating mutations. J. Thorac. Oncol. 9(6), 805-811 (2014).

23. Haspinger ER, Agustoni F, Torri V et al. Is there evidence for different effects among EGFR-TKIs? Systematic review and meta-analysis of EGFR tyrosine kinase inhibitors (TKIs) versus chemotherapy as first-line treatment for patients harboring EGFR mutations. Crit. Rev. Oncol. Hematol. 94(2), 213-227 (2015).

24. Liang W, Wu X, Fang W et al. Network meta-analysis of erlotinib, gefitinib, afatinib and icotinib in patients with advanced non-small-cell lung cancer harboring EGFR mutations. PLoS ONE 9(2), e85245 (2014).

25. Najafzadeh M, Schneeweiss S. From trial to target populations - calibrating real-world data. N. Engl. J. Med. 376(13), 1203-1205 (2017).

26. Lakdawalla DN, Shafrin J, Hou N et al. Predicting real-world effectiveness of cancer therapies using overall survival and progression-free survival from clinical trials: empirical evidence for the ASCO value framework. Value Health 20(7), 866-875 (2017).

27. Lau S, Chooback N, Ho C, Melosky B. P3.01-015 Differential outcomes between first and second generation TKIs in patients with activating EGFR mutations in NSCLC. J. Thorac. Oncol. 12(11), S2205 (2017).

28. Tu CY, Chen CM, Liao WC et al. Comparison of the effects of the three major tyrosine kinase inhibitors as first-line therapy for non-small-cell lung cancer harboring epidermal growth factor receptor mutations. Oncotarget 9(36), 24237-24247 (2018).

29. Kim Y, Lee SH, Ahn JS, Ahn MJ, Park K, Sun JM. Efficacy and safety of afatinib for EGFR-mutant non-small cell lung cancer, compared with gefitinib or erlotinib. Cancer Res. Treat. doi:10.4143/crt.2018.117 (2018).

30. Tung A, Hepp Z, Bansal A, Devine EB. Characterizing health care utilization, direct costs, and comorbidities associated with interstitial cystitis: a retrospective claims analysis. J. Manag. Care Spec. Pharm. 23(4), 474-482 (2017).

31. Divino V, Karve S, Gaughan A et al. Characteristics and treatment patterns among US patients with hairy cell leukemia: a retrospective claims analysis. J. Comp. Eff. Res. 6(6), 497-508 (2017).

32. Atzinger CB, Guo JJ. Biologic disease-modifying antirheumatic drugs in a national, privately insured population: utilization, expenditures, and price trends. Am. Health Drug Benefits 10(1), 27-36 (2017).

33. Xie L, Kariburyo F, Sah J et al. Real world medication use in incident systemic lupus erythematosus and lupus nephritis patients. Arthritis Rheumatol. 70(Suppl. 10), Abstract 2658 (2018).

34. Broder MS, Neary MP, Chang E et al. Treatments, complications, and healthcare utilization associated with acromegaly: a study in two large United States databases. Pituitary 17(4), 333-341 (2014).

35. Knight T, Bond TC, Popelar B et al. Medical resource utilization in dermatomyositis/polymyositis patients treated with repository corticotropin injection, intravenous immunoglobulin, and/orrituximab. CEOR 9, 271-279 (2017).

36. Joyce AT, Iacoviello JM, Nag S et al. End-stage renal disease-associated managed care costs among patients with and without diabetes. Diabetes Care 27(12), 2829-2835 (2004).

37. Paramore LC, Thomas SK, Knopf KB, Cragin LS, Fraeman KH. Estimating costs of care for patients with newly diagnosed metastatic colorectal cancer. Clin. Colorectal Cancer 6(1), 52-58 (2006).

38. Song X, Zhao Z, Barber B, Gregory C, Schutt D, Gao S. Characterizing medical care by disease phase in metastatic colorectal cancer. J. Oncol. Pract. 7(3 Suppl.), 25s-30s (2011).

39. Machado MA, Bernatsky S, Bessette L, Nedjar H, Rahme E. Hospitalization for musculoskeletal disorders in rheumatoid arthritis patients: a population-based study. BMC Musculoskelet. Disord. 17, 298 (2016).

40. Jalbert J, Gasse C, Bakshi $\mathrm{S}$ et al. Channeling bias in comparative-effectiveness research of newly launched medications: a case study. Value in Health 19(3), A184 (2016).

41. Ankarfeldt MZ, Thorsted BL, Groenwold RH, Adalsteinsson E, Ali MS, Klungel OH. Assessment of channeling bias among initiators of glucose-lowering drugs: a UK cohort study. Clin. Epidemiol. 9, 19-30 (2017).

42. De Groot V, Beckerman H, Lankhorst GJ, Bouter LM. How to measure comorbidity: a critical review of available methods. J. Clin. Epidemiol. 56(3), 221-229 (2003).

43. Deyo RA, Cherkin DC, Ciol MA. Adapting a clinical comorbidity index for use with ICD-9-CM administrative databases. J. Clin. Epidemiol. 45(6), 613-619 (1992).

44. Kettani FZ, Dragomir A, Cote R et al. Impact of a better adherence to antihypertensive agents on cerebrovascular disease for primary prevention. Stroke 40(1), 213-220 (2009)

45. Sajatovic M, Ng-Mak D, Solem CT, Lin FJ, Rajagopalan K, Loebel A. Dosing patterns and medication adherence in bipolar disorder patients treated with lurasidone: a US retrospective claims database analysis. Ther. Adv. Psychopharmacol. 6(6), 355-368 (2016). 
46. Pelletier EM, Smith PJ, Boye KS, Misurski DA, Tunis SL, Minshall ME. Direct medical costs for type 2 diabetes mellitus complications in the US commercial payer setting: a resource for economic research. Appl. Health Econ. Health Policy 6(2-3), 103-112 (2008).

47. Reynolds MW, Stephen R, Seaman C, Rajagopalan K. Persistence and adherence to disease modifying drugs among patients with multiple sclerosis. Curr. Med. Res. Opin. 26(3), 663-674 (2010).

48. Hung MS, Chen IC, Lee CP et al. Incidence and risk factors of depression after diagnosis of lung cancer: a nationwide population-based study. Medicine 96(19), e6864 (2017).

49. Brown CL, Hammill BG, Qualls LG, Curtis LH, Muir AJ. Significant morbidity and mortality among hospitalized end-stage liver disease patients in medicare. J. Pain Symptom Manag. 52(3), 412-419 e411 (2016).

50. Mooney JJ, Raimundo K, Chang E, Broder MS. Mechanical ventilation in idiopathic pulmonary fibrosis: a nationwide analysis of ventilator use, outcomes, and resource burden. BMC Pulm. Med. 17(1), 84 (2017).

51. Henk HJ, Ray S. Treatment patterns and healthcare costs among patients with advanced non-small-cell lung cancer. Lung Cancer Manag. 2(3), 189-197 (2013).

52. Yonesaka K, Kudo K, Nishida S et al. The pan-HER family tyrosine kinase inhibitor afatinib overcomes HER3 ligand heregulin-mediated resistance to EGFR inhibitors in non-small cell lung cancer. Oncotarget 6(32), 33602-33611 (2015).

53. Yu HA, Arcila ME, Rekhtman N et al. Analysis of tumor specimens at the time of acquired resistance to EGFR-TKI therapy in 155 patients with EGFR-mutant lung cancers. Clin. Cancer Res. 19(8), 2240-2247 (2013).

54. Miller VA, Hirsh V, Cadranel J et al. Afatinib versus placebo for patients with advanced, metastatic non-small-cell lung cancer after failure of erlotinib, gefitinib, or both, and one or two lines of chemotherapy (LUX-Lung 1): a Phase IIb/3 randomised trial. Lancet Oncol. 13(5), 528-538 (2012).

55. Katakami N, Atagi S, Goto K et al. LUX-Lung 4: a Phase II trial of afatinib in patients with advanced non-small-cell lung cancer who progressed during prior treatment with erlotinib, gefitinib, or both. J. Clin. Oncol. 31(27), 3335-3341 (2013).

56. Schuler M, Yang JC, Park K et al. Afatinib beyond progression in patients with non-small-cell lung cancer following chemotherapy, erlotinib/gefitinib and afatinib: Phase III randomized LUX-Lung 5 trial. Ann. Oncol. 27(3), 417-423 (2016).

57. Ito K, Murotani K, Kubo A et al. Comparative analysis of overall survival using propensity score between first- and second-generation EGFR-TKI: real world data of 1354 patients with EGFR mutant NSCLC. Ann. Oncol. 29(Suppl. 8), viii493-viii547 (2018).

-. Analysis investigating time on treatment with afatinib, erlotinib or gefitinib in a large cohort of patients with EGFR mutation-positive NSCLC treated in Japan.

58. Hochmair MJ, Morabito A, Hao D et al. Sequential treatment with afatinib and osimertinib in patients with EGFR mutation-positive non-small-cell lung cancer: an observational study. Future Oncol. 14(27), 2861-2874 (2018).

59. Halmos B, Tan E-H, Soo RA et al. Impact of afatinib dose modification on safety and effectiveness in patients with EGFR mutation-positive advanced NSCLC: results from a global real-world study (RealGiDo). Lung Cancer 127, 103-111 (2019).

60. Hess LM, Louder A, Winfree K, Zhu YE, Oton AB, Nair R. Factors associated with adherence to and treatment duration of erlotinib among patients with non-small cell lung cancer. J. Manag. Care Spec. Pharm. 23(6), 643-652 (2017).

61. Fukuoka M, Wu YL, Thongprasert $S$ et al. Biomarker analyses and final overall survival results from a Phase III, randomized, open-label, first-line study of gefitinib versus carboplatin/paclitaxel in clinically selected patients with advanced non-small-cell lung cancer in Asia (IPASS). J. Clin. Oncol. 29(21), 2866-2874 (2011).

62. Inoue $\mathrm{A}$, Kobayashi $\mathrm{K}$, Maemondo $\mathrm{M}$ et al. Updated overall survival results from a randomized Phase III trial comparing gefitinib with carboplatin-paclitaxel for chemo-naïve non-small cell lung cancer with sensitive EGFR gene mutations (NEJ002). Ann. Oncol. 24(1), 54-59 (2013).

63. Sequist L, Wu YL, Schuler M et al. 1349P Subsequent therapies post-afatinib among patients (pts) with EGFR mutation-positive $($ EGFRm+) NSCLC in LUX-Lung (LL) 3, 6 and 7. Ann. Oncol. 28(Suppl. 5), mdx380.051-mdx380.051 (2017). 